\title{
Trabecular bone deterioration at the greater trochanter of mice with unilateral obstructive nephropathy
}

\author{
Sa-Sa Gu ${ }^{1}$, Yan Zhang ${ }^{1,2,3}$, Xi Chen ${ }^{2}$, Teng-Yue Diao ${ }^{2}$, Yoseph Gebru ${ }^{2}$ and Man-Sau Wong ${ }^{3}$
}

Our previous study showed the early molecular responses of bone in response to obstructive nephropathy in a unilateral ureteral obstruction (UUO) mouse model. Here, we addressed the changes in trabecular bone properties at greater trochanter, the proximal and the distal metaphysis of femur in UUO mice. The male mice were subjected to UUO $(n=10)$ or sham operation $(n=10)$. All mice were killed on day 7 after the surgical operation. The micro-computed tomography (micro-CT) analysis for different femoral trabecular bone sites demonstrated pathological alterations of trabecular bone mass and micro-networks at greater trochanter as shown by decreases in bone mineral density/bone volume $(\boldsymbol{P}<0.05)$ and trabecular number $(\boldsymbol{P}<0.05)$ and increases in trabecular separation $(P<0.01)$ and bone surface/bone volume $(P<0.05)$ in UUO mice. The present study demonstrates that UUO-induced unilateral obstructive nephropathy has markedly detrimental effects on the trabecular trochanter of the femur.

Asian Journal of Andrology (2013) 15, 564-566; doi:10.1038/aja.2013.34; published online 6 May 2013

Keywords: bone; femur; micro-computed tomography (micro-CT); trochanter; unilateral ureteral obstruction (UUO)

\section{INTRODUCTION}

Changes in bone morphology and histology, such as variations in turnover $(\mathrm{T})$, bone mineralisation $(\mathrm{M})$ and volume $(\mathrm{V})$ and vascular or other soft-tissue calcifications, have been included under the definition of 'chronic kidney disease-mineral and bone disorders' (CKDMBD). ${ }^{1}$ The TMV system provides relevant information and is widely used to evaluate CKD patient bone health; ${ }^{2}$ however, this information should be expanded to include cancellous bone architecture.

Unilateral ureteral obstruction (UUO) induces damage in the unilateral obstructed kidney and is a well-established model of tubulointerstitial kidney fibrosis. ${ }^{3}$ We previously reported changes in the renal expression of vitamin D metabolic enzymes and changes in calcium transporter abundance following obstructive nephropathy in mice subjected to $\mathrm{UUO}^{4}$ and demonstrated early molecular responses of bone to unilateral obstructive nephropathy in the UUO model. ${ }^{5}$ However, associated with the pathological alterations of vitamin D metabolism and the expression of genes involved in bone metabolism, whether trabecular bone microstructure would deteriorate in UUO mice remained to be clarified.

The availability of three-dimensional (3D) measuring techniques opens new avenues to analyse the biological properties of bone. In this study, femoral trabecular bone parameters were determined by microcomputed tomography (micro-CT) to qualitatively illustrate the alterations of bone properties in mice that were subjected to unilateral obstructive nephropathy.

\section{MATERIALS AND METHODS}

Animal studies

Two-month-old ICR male mice (Slac Laboratory Animal, Shanghai, China) were fed a commercial diet for 1 week during acclimatisation before being subjected to either sham operation or UUO. Briefly, UUO $(n=10)$ was performed under pentobarbital sodium $\left(50 \mathrm{mg} \mathrm{kg}^{-1}\right.$; Sigma, St Louis, MO, USA) anaesthesia. A midline incision was made, and the left ureter was exposed and tied off at two points. Shamoperated mice $(n=10)$ were used as controls. Mice in both groups were killed on day 7 after the surgical operation by cardiac exsanguination under light ether anaesthesia. The femurs were wrapped in salinesoaked towels and stored at $-20^{\circ} \mathrm{C}$ until further analysis. The animal study protocols were approved by the Department of Research Administration, and all experiments were conducted according to the guidelines of the Animal Welfare Committee of the University of Shanghai for Science and Technology.

\section{Micro-CT analysis}

The whole femur of each animal was scanned to assess image, and then the proximal and distal femoral metaphysis and trochanteric sites were examined as 1.165 and $0.493 \mathrm{~mm}$ slabs, corresponding to 111 and 47 slices, respectively, with a high-resolution micro-vivaCT 40 system (Scanco Medical, Bassersdorf, Switzerland). Trabecular bone was determined by a fixed threshold. Hand-drawn contours were used to isolate the regions of interest and trabecular compartments based on 30 consecutive slices applied to the trabecular trochanter and 50 to the proximal metaphysis and 60 to the distal end of femur. Bone microarchitecture was assessed with direct 3D methods using the $\mu \mathrm{CT}$ Evaluation Program (Image Processing Language v. 5.0A; Scanco Medical). Morphological 3D parameters for trabecular bone were obtained as follows: (i) bone volume/total volume (BV/TV), (ii) structure model index (SMI), (iii) trabecular number (Tb.N), (iv) 
trabecular thickness (Tb.Th), (v) trabecular separation (Tb.Sp), (vi) mean mineral density of bone volume (BMD/BV) and (vii) bone surface per given bone volume (BS/BV). Additionally, 3D images were reconstructed for selected consecutive slices.

\section{Statistical analysis}

Data are presented as mean \pm s.e.m. Statistical differences were analysed by $t$-test, with $P<0.05$ considered to be statistically significant.

\section{RESULTS}

To illustrate trabecular bone property changes in the femurs of mice following unilateral obstructive nephropathy, three different bone sites, including the greater trochanter and proximal and distal metaphysis, were assessed by micro-CT as shown in Figure 1. The 3D and 2D images (Figure 2) clearly show osteopaenia in the trabecular trochanter of UUO mice (Figure $\mathbf{2 b}, \mathbf{d}$ ), and these pathological changes were quantitatively demonstrated by the $3 \mathrm{D}$ bone parameters (Table 1).

Compared to the corresponding parameters in the controls, unilateral obstructive nephropathy triggered significant decreases in the structure model index (by $64.5 \% ; P<0.01$ ), the Tb.N (by $13.4 \%$; $P<0.05$ ) and BMD/BV (by $10.4 \% ; P<0.05)$ and caused a corresponding increase of $25 \%(P<0.01)$ in Tb.Sp and of $30 \%(P<0.05)$ in BS/BV at the greater trochanter. Notably, only the SMI values at the proximal and distal femoral metaphysis were significantly reduced in UUO mice.

\section{DISCUSSION}

This study demonstrated that compared to other femoral trabecular bone sites, the trochanter site was sensitive to unilateral obstructive nephropathy induced by UUO. Loss of trabecular bone mass at the greater trochanter was shown in UUO mice, which is in accordance with the clinical observation of intertrochanteric fracture with bilateral avulsion of the greater trochanter in a patient with chronic renal failure $^{6}$ and significantly decreased BMD of the greater trochanter in CKD patients. ${ }^{7}$ Moreover, the present study revealed trabecular bone network deterioration with micro-CT data (Table 1) and the reconstructed $2 \mathrm{D}$ and $3 \mathrm{D}$ images (Figure 2). In addition, the potential increase in bone resorption in the femoral greater trochanter reflected

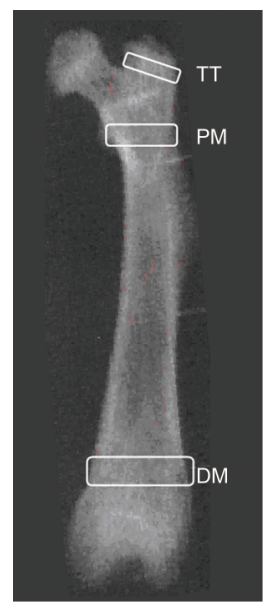

Figure 1 Femoral trabecular bone sites measured by micro-CT. CT, computed tomography; DM, distal metaphysis; PM, proximal metaphysis; TT, trabecular trochanter.

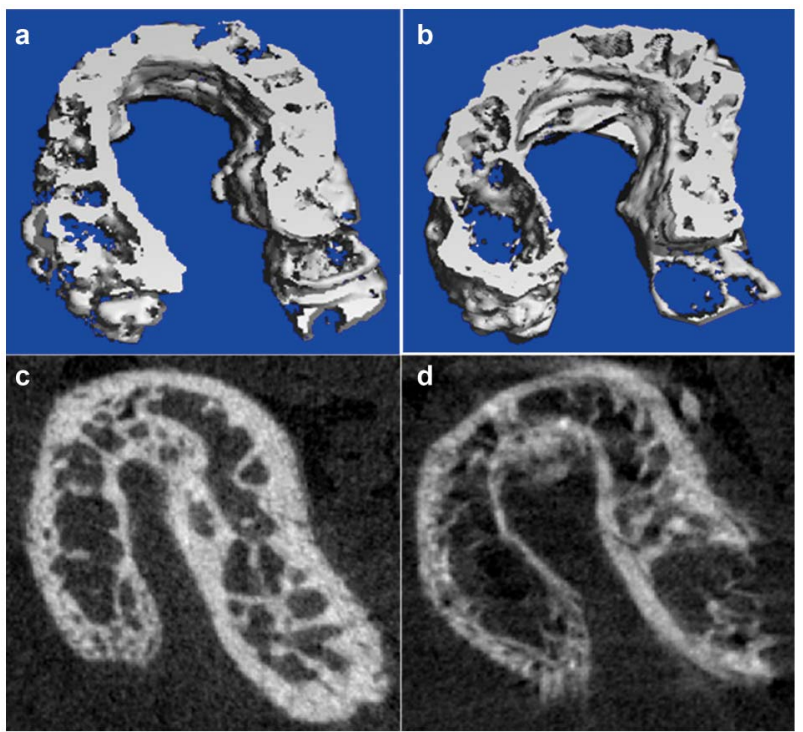

Figure 2 Three-dimensional (a, b) and two-dimensional (c, d) images of greater trochanter trabecular bone analysed by micro-CT in the control group (a, c) and UUO group (b, d). CT, computed tomography; UUO, unilateral ureteral obstruction.

by BS/BV may, at least partially, contribute to bone deterioration in UUO mice.

SMI is a parameter that can be used to quantify the 'rod-like' or 'plate-like' architecture. ${ }^{8}$ The SMI values for parallel plates and cylindrical rods are 0 and 3, respectively. ${ }^{9}$ According to the SMI values in this study, trabecular bone tested at all three femoral sites of the UUO group had a more pronounced plate structure than that of the control group, and the reason for this outcome requires further examination. Additionally, the mechanical strength of the femoral trabecular bone associated with alterations of bone mass and structure during unilateral obstructive nephropathy should be investigated in future studies because bone mechanics depend on both the structural model and apparent density. ${ }^{10}$

Altogether, our present study clearly indicates that the greater trochanter is a sensitive bone site that exhibits loss of bone mass and trabecular bone network damage in response to unilateral obstructive nephropathy. As we previously reported, the underlying mechanism may, at least partially, be due to the increased activity of the local renin-angiotensin system in bone and the pathological changes in the serum levels of minerals and parathyroid hormone in UUO mice. ${ }^{5}$ The present results suggest that clinical bone health monitoring of the femoral greater trochanter might need to be performed for CKD patients, especially those with unilateral obstructive nephropathy.

\section{AUTHOR CONTRIBUTIONS}

YZ and MSW developed the study protocol and the experimental design, SSG, XC, TYD and YG collected samples and conducted experiments, SSG and XC analysed data, YZ wrote the draft of the manuscript and MSW reviewed the final manuscript. All authors read and approved the final manuscript.

\section{COMPETING FINANCIAL INTERESTS}

None of the authors had any personal or financial conflicts of interest. 
Table 1 Trabecular bone parameters at the trochanter, proximal metaphysis and distal metaphysis of the femur as detected by micro-CT

\begin{tabular}{|c|c|c|c|c|c|c|c|c|}
\hline & & $B V / T V$ & $S M I$ & Tb.N (1 per $\mathrm{mm})$ & Tb.Th $(\mu \mathrm{m})$ & $T b . S p(\mu m)$ & $\begin{array}{c}B M D / B V \\
\text { (mg HA per ccm) }\end{array}$ & $B S / B V(1$ per $m m)$ \\
\hline \multirow[t]{2}{*}{ Trochanter } & Control & $0.46 \pm 0.05$ & $1.83 \pm 0.24$ & $14.65 \pm 0.20$ & $57.2 \pm 1.9$ & $77.6 \pm 1.8$ & $723 \pm 12$ & $8.3 \pm 0.7$ \\
\hline & UUO & $0.52 \pm 0.05$ & $0.65 \pm 0.23^{* *}$ & $12.68 \pm 0.57 *$ & $54.6 \pm 1.5$ & $96.8 \pm 4.0^{* *}$ & $648 \pm 27 *$ & $10.8 \pm 0.2^{*}$ \\
\hline Proximal metaphysis & UUO & $0.43 \pm 0.01$ & $0.88 \pm 0.30 * *$ & $10.75 \pm 0.19$ & $70.4 \pm 3.0$ & $106.5 \pm 4.0$ & $726 \pm 16$ & $6.4 \pm 0.1$ \\
\hline \multirow[t]{2}{*}{ Distal metaphysis } & Control & $0.53 \pm 0.06$ & $2.17 \pm 0.36$ & $9.40 \pm 0.62$ & $79.5 \pm 6.9$ & $105.4 \pm 8.3$ & $704 \pm 17$ & $6.8 \pm 0.5$ \\
\hline & UUO & $0.52 \pm 0.02$ & $1.41 \pm 0.27 *$ & $9.78 \pm 0.55$ & $73.0 \pm 4.5$ & $99.5 \pm 8.0$ & $676 \pm 16$ & $7.3 \pm 0.3$ \\
\hline
\end{tabular}

Abbreviations: BMD/BV: volumetric bone mineral density; BS/BV: ratio of segmented bone surface to segmented bone volume, indicating the resorption area by osteoclasts; $\mathrm{BV} / \mathrm{TV}$ : ratio of the segmented bone volume to the total volume of the region of interest, indicating the fraction of a given volume of interest occupied by mineralized bone; $\mathrm{CT}$, computed tomography; SMI: an indicator of trabecular bone structure, 0 for parallel plates and 3 for cylindrical rods; Tb.N: measure of the average number of trabeculae per unit length; Tb.Th: mean trabeculae thickness; Tb.Sp: mean distance between trabeculae. Tb.N, Tb.Th and Tb.Sp are normally used for evaluating trabecular bone microarchitecture; UUO, unilateral ureteral obstruction.

Values are expressed as mean \pm s.e.m., $n=10$ in each group.

$* P<0.05, * * P<0.01$, compared to the control group.

\section{ACKNOWLEDGMENTS}

This work was sponsored by the Innovation Program of Shanghai Municipal Education Commission (No. 11ZZ137) and the Shanghai Pujiang Program (No. 10PJ1407700) for Yan Zhang. It was partially supported by the Hong Kong Scholars Program (No. XJ2011022) and the China Postdoctoral Science Foundation (No. 2012M511115).

1 Mejía N, Roman-García P, Miar AB, Tavira B, Cannata-Andía JB. Chronic kidney diseasemineral and bone disorder: a complex scenario. Nefrologia 2011; 31: 514-9.

2 Malluche HH, Mawad HW, Monier-Faugere MC. Renal osteodystrophy in the first decade of the new millennium: analysis of 630 bone biopsies in black and white patients. J Bone Miner Res 2011; 26: 1368-76.

3 Zhang Y, Kong J, Chang A, Deb DK, Li YC. Vitamin D receptor attenuates renal fibrosis in obstructive nephropathy by suppressing the renin-angiotensin system. J Am Soc Nephrol 2010; 21: 966-73.
4 Zhang Y, Wu SY, Gu SS, Lv FK. Changes of renal vitamin D metabolic enzyme expression and calcium transporter abundance in obstructive nephropathy. Nephrology 2011; 16: 710-4.

5 Gu SS, Zhang Y, Wu SY, Diao TY, Gebru YA et al. Early molecular responses of bone to obstructive nephropathy induced by unilateral ureteral obstruction in mice. Nephrology 2012; 17: 767-73.

6 Akçali O, Koşay C, Günal I, Alici E. Bilateral trochanteric fractures of the femur in a patient with chronic renal failure. Int Orthop 2000; 24: 179-80.

7 Jiang JQ, Lin S, Xu PC, Zheng ZF, Jia JY. Serum osteoprotegerin measurement for early diagnosis of chronic kidney disease-mineral and bone disorder. Nephrology2011; 16: 588-94.

8 Zhang $\mathrm{Y}$, Li Q, Li XL, Wan HY, Wong MS. Erythrina variegata extract exerts osteoprotective effects by suppression of the process of bone resorption. Br J Nutr 2010; 104: 965-71.

9 Bouxsein ML, Boyd SK, Christiansen BA, Guldberg RE, Jepsen KJ et al. Guidelines for assessment of bone microstructure in rodents using micro-computed tomography. $J$ Bone Miner Res 2010; 25: 1468-86.

10 Hildebrand T, Rüegsegger P. Quantification of bone microarchitecture with the structure model index. Comput Methods Biomech Biomed Engin 1997; 1: 1523. 